\title{
Smell Impregnated Images, Encoding, Generation and Its Implications
}

\author{
Saad Hameed Abid ${ }^{1,2}$, Zhiyong $\mathrm{Li}^{1}$, Renfa $\mathrm{Li}^{1}$ and Jumana Waleed ${ }^{3}$ \\ ${ }^{1}$ College of Computer Sciences and Electronic Engineering, \\ Hunan University, Changsha, Hunan 410082, China \\ saad@hnu.edu.cn,zhiyong.li@hnu.edu.cn, \\ lirenfa@hnu.edu.cn \\ ${ }^{2}$ AL-Mansour University College, Al-Andalus square, Baghdad, Iraq \\ ${ }^{3}$ School of information science and engineering, Central South University, \\ Changsha, 410083, China \\ jumana@csu.edu.cn
}

\begin{abstract}
Recently, many types of media files have joined the multimedial family not to mention the significant improvements on the existing ones. Furthermore there are other extensive researches regarding olfactory displays or smell generators, these devices are capable of generating different intensities of smells, yet still has some limitations and not yet set for commercial use. In this work, a complete solution were presented to enable images to contain smell information for the user to experience another depth of interaction with olfaction sensation along with the auditory, haptic and visual. Using a novel olfactory display device based on a micro-porous piezoelectric film that is capable of producing very fine particles of the scent material with precision that is almost digitally in term of amount and speed.
\end{abstract}

Keywords: Smell, olfactory displays, piezoelectric, image smell

\section{Introduction}

Many researches has been done regarding olfactory displays yet instead of focusing on building the display itself and all the implications and challenges that might arise, it is better to focus on both introducing a novel approach of smell generation and a robust way to embed this smell information into images, the delivery is achieved using a piezoelectric transducer film with very fine mesh to generate a droplet so little that they can be controlled digitally; providing a discrete amount (digital) of smell keeping in mind that the smell should travel from the container holding that smell to the nose of the user in minimal time which is in a matter of a second or less.

\section{Related Works}

There are a considerably significant amount of researches done in the field of olfactory displays using many technologies to provide a better and fast delivery of scented material to the user, these attempts have good and successful results yet incomplete and have some drawbacks. The problem in focus is how to: First, successfully build a scent providing element that is capable of transforming the scented material state (mostly liquid) to a state can be intercepted by human olfactory display (air borne molecules). Second: provide a sound mechanism to digitize the concentration of the scented material. Third: have a response time sufficient to the application being used putting in consideration the human's olfactory system response to the projected scent, it is important to state that these 
attempts considered the foundation of olfactory displays yet in [1] the generation of scent material depends on applying a current to peltier module that the scent element is placed on top of it, this enables the olfactory display to have more than one scent element but the peltier module converts current to heat to evaporate the scented material which requires significant amounts of both voltage and current in addition to heating time which is a critical factor in olfactory displays. Also in [2] it uses a micro pump to deliver the scented material onto a SAW (surface acoustic wave) device it has promising results but the hardware setup is not practical and requires voltage levels as high as 100volts for the driving circuit. Other olfactory displays such as [3] and [4] use solenoids valves to control the flow of the scented material and/or air flow, since the solenoids are electromagnetic devices with mechanical movement they require a significant amount of energy and produces heat and clanking noise while operating not to mention the air pump that providing the air flow to the olfactory display. In [5] a more evolving model of the olfactory display that is actually included into an application, in this case sensing the smell into a certain device and sending primitive olfactory data merged with the camera captured image through ADSL connection via internet in fact Nakamoto, et al., has the most maturing researches in the field of olfactory displays but the smell projection device they used is based on [3]. The claim is that a complete system for an olfactory display actually used both practically and commercially that satisfies compactness, efficiency, durability, speed and user friendly which does not require an engineer or scientist to maintain and operate the device and also does not require a lab and other external equipment and special environment to successfully use it, simply a user and a PC with a selection of any aromatic material (water or oil based) that the user desires or the image content/nature dictates.

\section{Olfactory Display's Design}

A micro-porous piezoelectric film was used in the proposed olfactory display that in essence a piezoelectric film with a steel mesh in the center of the transducer, when connected to a certain frequency it will vibrate creating a very small sized air born droplets that projected to the users' nose.

\subsection{Scent Ejection Cartridge}

Using a piezoelectric film with micro sized holes in the center, mounted in front of a transparent plastic cartridge with a silicone washer to prevent leakage of the scented material which cause inaccurate results; the plastic cartridge has two opposite openings one is used to mount the piezoelectric film to extrude the scented material and the other hole is used to fill the cartridge with the scented material. Two silicone seal covers (upper and lower) are placed to create a sandwich enclosing the piezoelectric film, this sandwich is fastened under the scent cartridge using tiny screw nails to create an air tight chamber, this will prevent the scented material from leaking into the olfactory display in any form (vapor or liquid), see Figure 1, a diagram of the scent element cartridge assembly. 


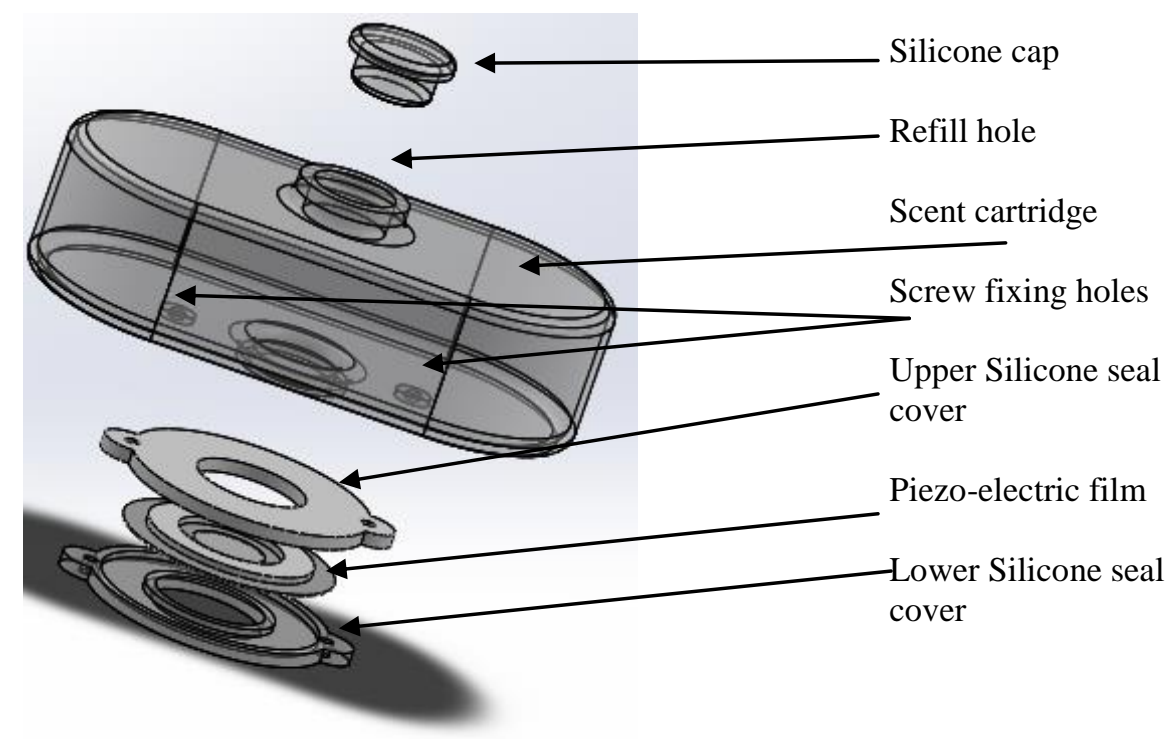

Figure 1. The Scent Element Assembly with Scented Material Cartridge and All the Upper and Lower Caskets Using Two Screw Nails to Hold the Assembly Tight and creating a Durable Component

The piezoelectric film is enclosed inside of the upper and lower silicone caskets leaving a small gap between the film and the silicon, the film will vibrate due to the supplied frequency causing the scented material to be extruded out of the micro sized holes by the front action due to the positive phase of the signal and in the back action due to the opposite phase of the signal repelling the scented material creating a micro sized air born droplet that rushing out of the scent element, shown later in Figures 3 and 4 for the operation cycle of the scent production material.

The Atomization piezoelectric film or the micro porous piezoelectric film has the following characteristics:

1) Diameter: $13.8 \mathrm{~mm}$.

2) Low driving voltage: $3-12 \mathrm{~V}$.

3) High conversion efficiency, spray volume.

4) Exit aperture is very small $4 \mu \mathrm{m}$.

5) Frequency: $113 \mathrm{khz} \pm 5 \mathrm{khz}$.

6) Capacitance: $2700 \mathrm{PF} \pm 15 \%$.

7) Power: 1.5-2.0W.

8) Spray volume $30 \mathrm{ml} / \mathrm{h}$.

9) Can atomize essential oils, perfume, water based perfumes or even mixture of the above materials.

10) Life of more than 3000 hours.

Noticing that most its characteristics is suitable to be used in computer applications that requires low energy consumption and high efficiency operation, the size of the droplet is about $4 \mu \mathrm{m}$ which is small enough to travel air born, the movement of the atomizer is continuous to produce more and more of these droplets forcing them to be repelled from the exit hole of the scent delivery cartridge and into the mixing chamber of the olfactory display, as long as the input signal is continuous then the fine mist production is also continuous, Figure 2 is the implementation of six scent elements that are used in the proposed olfactory display. 


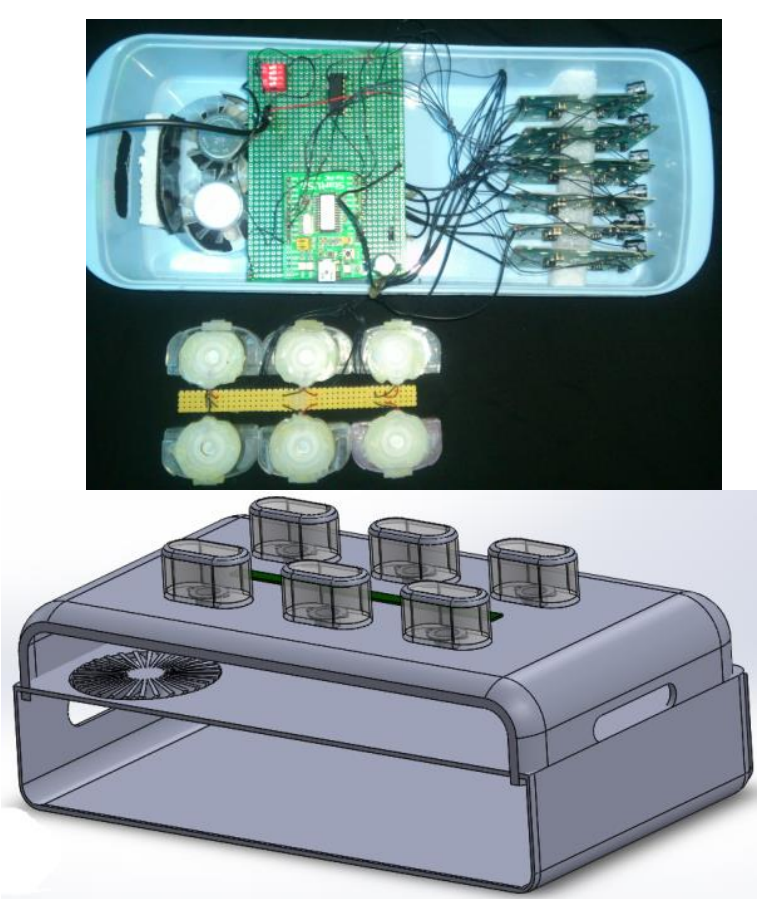

Figure 2. An Implementation of Six Scent Element Cartridges Connected via Circuit Board and Wired by $0.5 \mathrm{~mm}$ to the Olfactory Display's Frequency Generator Boards

As seen in the characteristics of the transducer, the input frequency is rather high and an external circuit is needed built to produce this frequency which essential to the activation of the transducer film to produce the required mist volume, this frequency ranges from $108 \mathrm{kHz}$ to $118 \mathrm{kHz}$, In the early experiments an attempt to manipulate the transducers' supplied frequency to control the amount of the generated scent material.

In some sense it was successful, but with not much noticeable variation, if the frequency is reduced to $68 \%$ or less, then the droplets are not produced correctly just a single cluttered drops falling off into the mixing chamber, if the frequency is higher than $110 \%$ the droplets are also deformed and not produced correctly. For the values in between $68 \%-90 \%$ has no noticeable difference in the volume of produced mist, as a result timed pulses were used to produce required scent intensities instead of altering the applied frequencies.

As mentioned before that the operation of the transducer film requires a frequency, that frequency essentially will make the film vibrated into the same amount of times that the frequency changes, the film consists of two plates that bends back and forth according to the frequency variation of the supplied frequency. When the value of the supplied signal is zero then the plate is in a "resting" state and produces no droplets or mist, in addition to that, the holes in the center is so small that it prevents the liquid material to pass through it, see Figure 3. 


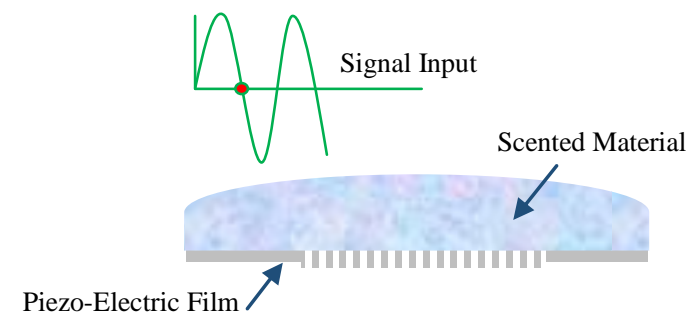

\section{Figure 3. Shows a Piezoelectric Mesh Atomizer Film in Resting State Noticing that the Small Sized Holes dose not Leak Scent}

As the voltage starts to drop into the negative region of the voltage level, the piezoelectric film will bend upwards creating a pressure inside the cartridge, in addition, the sudden contraction creates a force on the scented material directly surrounding the micro holes to be forced thought the holes, again, these holes are too small which will squirt the scented material through that holes in very small amounts $(6 \mu \mathrm{L})$, which produces a fine droplets, see Figure 4(a). Afterwards, when the signal starts to rise again into the positive region of the voltage level, the piezoelectric transducer starts to bend outwards, and since this happens very quickly (after the upwards bending) then the accumulated fine droplets are repelled and forced to find its direction away from the micro holes allowing the next droplets to be produced freely and the old droplets to gain momentum to move away from the piezoelectric transducer, see Figure 4(b).

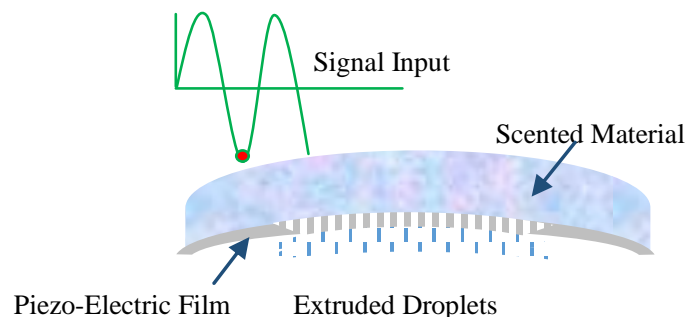

(a)

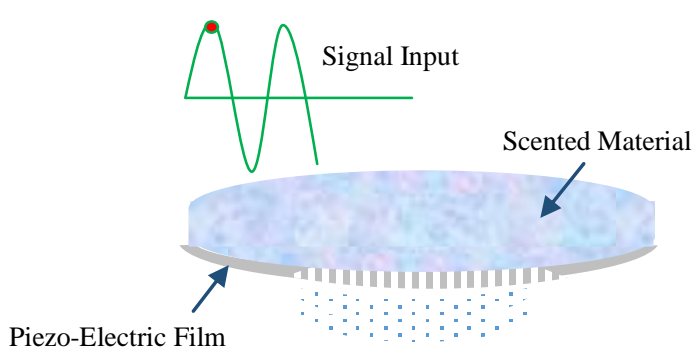

Ejected Droplets

Figure 4. (a) Shows a Piezoelectric Mesh Atomizer Film in Upward Bend State Extruding Micro Droplets in the Process, (b) Shows a Piezoelectric Mesh Atomizer Film in Downward Bend State which Forces the Droplets to Move Away from the Film to repeat the Process Again

\subsection{Data Generation of Scented Information}

In this component the user can set and modify the scent data that are going to be embedded in the specific image in target, the setting is done by clicking the timeline associated with the image or by setting duration of time for that scented data to be presented. The interface contains six channels with six different scent intensities for each channel, each intensity is marked through the image timeline with its own channel indicated by a distinctive color so the user can notice the scent information intensity level and its location, then the proposed system generates a corresponding binary image patterns each of which contains a different dither density.

Using dithering, six different intensities of each scent data were created, this is essential to withstand a convenient level of robustness for the scent data to persist throughout different attacks such as noise and compression, see Figure 5, although a breakthrough has been achieved in term of robustness were concerning the embedding process but the focus is not on that issue, the focus was making sure that the data will be 
extracted successfully in the proposed olfactory based image viewer, the final embedded data is constructed as a single binary bitmap with six region each region represent a single data instruction that corresponds to a particular scent element, of course if the image (region) does not contain a scent data then the image (region) will contain solid white color meaning the scent element will remain in active during the display of that frame.

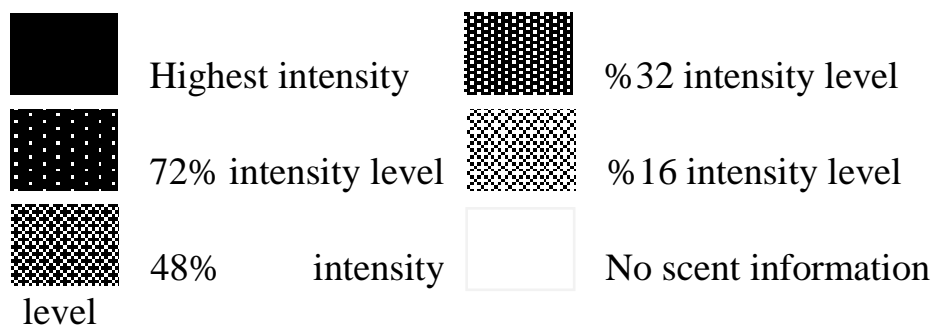

\section{Figure 5. Proposed Six Levels of Intensity Using Black Pixels Dithering Over White Background, where the Pixel Amount}

The generated bitmapped image is shown in Figure 6, with these six regions each of which carries the scent intensity to the corresponding scent element, and furthermore a stripe of half sized region contains all the intensity levels used in the system to use it later in the qualification process of extracted scent bitmap. This bitmapped image is embedded in the image to carry the scent intensity information as well as the selection of the scent element itself.

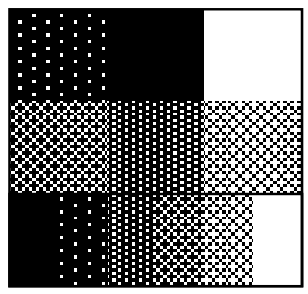

\section{Figure 6. Proposed Bitmap Image with Six regions of Intensity for Each Scent Element (Density) represents the Intensity Level of that Bitmap} Region

\subsection{Scent Data Embedding Engine}

The embedding system uses the blue channel to embed the actual data (scent data) because of the blue color variation is hardly perceived by the human visual system. For embedding the scent bitmap image, a Discrete Wavelet Transform (DWT) is performed to the blue channel. Scent bitmap information is embedded in the high frequency bands $(H H)$ of that channel since it includes the edges and textures of the image and the human eye is not generally sensitive to changes in such sub bands, also it is robust against various image processing operations and attacks. The following steps show the embedding process [6]:

Step1: Reformulate the binary bitmap image into a vector $W=\left\{w_{1}, w_{2}, \ldots, w_{m}\right\}$ of binaries.

Step2: Extract the blue channel from the RGB image and decompose it into DWT with one-level.

Step3: Split the $H H$ and divide it into non-overlapping blocks of dimension $8 \times 8, B_{1}, B_{2}$, $\ldots, B_{b}$. where $b$ is the number of blocks.

Step4: Apply the binary image bits $W$ into $H H$ blocks. The embedding process for each image bit is done by replacing the first coefficient in the first column in the block with the maximum coefficient $(M A X I M U M+\delta)$ in the same column if the embedded bit equal to 
1, else the replacement is done with the minimum coefficient (MINIMUM $-\epsilon)$, where $\delta$ and $\epsilon$ are the diversity strength factor parameters for the embedded image.

For each block $B_{i}$ :

$$
\begin{aligned}
& B_{i}{ }^{\prime}(1,1)=\operatorname{MAXIMUM}\left(B_{i}(r, 1)\right)+\delta \text { if } W_{i}=1 \text {, } \\
& B_{i}^{\prime}(1,1)=\operatorname{MINIMUM}\left(B_{i}(r, 1)\right)-\epsilon \quad \text { if } W_{i}=0 \text {. }
\end{aligned}
$$

Step5: Reconstruct the embedded blocks into $H H$ and perform inverse DWT to obtain the embedded blue channel, and combine the blue channel with the red and green channels to construct the smell impregnated image. The overall process of the embedding algorithm is showed in Figure 7.

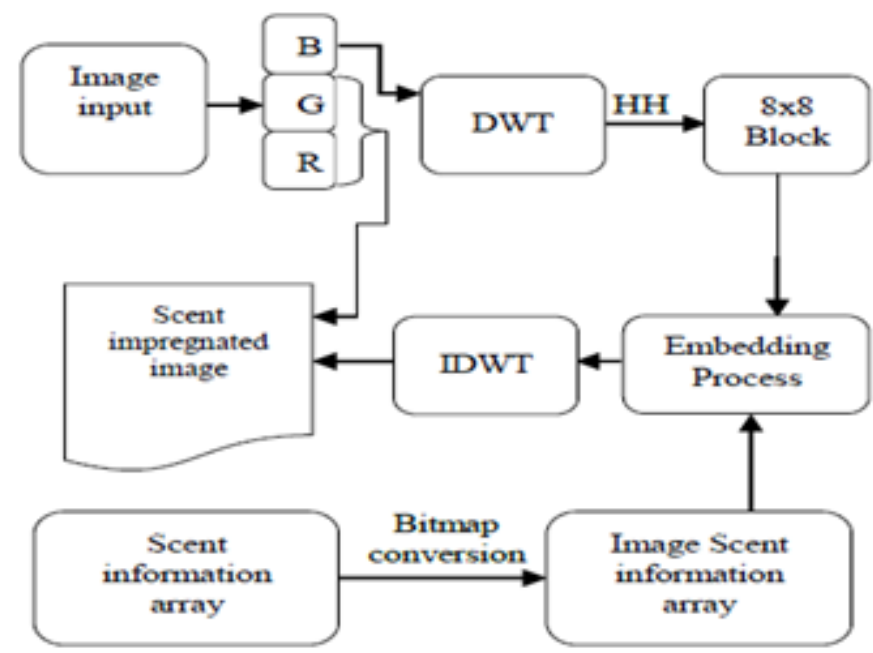

\section{Figure 7. A Block Diagram Explaining the Proposed Embedding Algorithm to Embed Scent Information Bitmap into Blue channel of Image File Using DWT}

\subsection{Olfactory Based Image Viewer}

This software component uses the image resulting from the embedding process and displays the image information along with the scent data, the viewer starts by extracting scent data information from the image creating a scent data array synchronized by the prior information from the user or providing the smell as one output after mixing these scent in the olfactory display's mixing chamber, when the viewer displays a specific image, it simultaneously sends the associated scent data to the proposed olfactory display via USB connection using a six bytes to carry out the data just for the scent information.

In the extraction process the proposed system extracts the embedded informational image according to the steps below and starts to calculate the density of one's (black pixels) in $48 \times 48$ image region and qualifies these values according to a qualification region threshold to successfully decide the amount of scent material to be produced corresponding to that image, the embedded image is very immune to noise because it doesn't carry neither visual nor discrete information rather that it carries statistical appearance of ones and zeros into a specific region, this statistical information or let's call it a density value that its difference property remains static even though their values changes rapidly.

The original image is not required during the extraction process, for successful extraction of the embedded scent bitmap, see Figure 8.

Step1: Extract the blue channel from the RGB embedded image and decompose it into DWT with one-level.

Step2: Split the $H H$ and divide it into blocks of dimension $8 \times 8$.

Step3: The extraction process is done by computing the average of the first column of each embedded block, and if the average is greater than the first coefficient in the first 
column then the embedded bit is equal to zero, otherwise the embedded bit is equal to one.

For each block Bi':

$$
\begin{aligned}
& W i^{\prime}=1, \text { if } B i^{\prime}(1,1)>=A V E R A G E, \\
& (2) \\
& W i^{\prime}=0, \text { if } B i^{\prime}(1,1)<A V E R A G E .
\end{aligned}
$$

Step4: The image contains scent data whether white bitmap for no scent data or some arbitrary data contained in it, these bitmaps regions are qualified according to qualification process to verify their values due to noise and some attacks (the qualification process will be explained later in this paper).

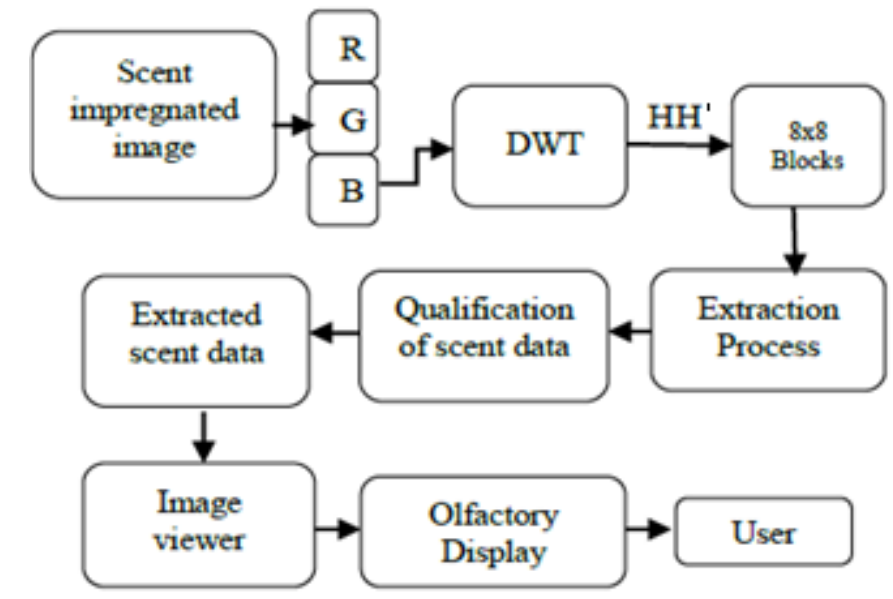

Figure 8. A Block Diagram Explaining the Proposed Extraction Algorithm to Extract Scent Information Bitmap from the Image File using DWT

\subsection{Extracted Scent Data Validation}

As the bitmap extracted successfully there are some issues need to be dealt with, such as noise and data deformation, in perfect conditions the extracted scent bitmap is not affected by the variations of compressions, noise and other affecting processes, but unfortunately in the real world this is a rare condition, since the intent is to maintain the scent information throughout the entire lifetime of the image file, as a result each region is checked against a threshold indicating that region level of intensity, the usage of dithering process gives the scent bitmap a high robustness against virtually all types of attacks.

The stripe of intensities (qualification region) is used as a guideline for the threshold construction and its values will be affected by the same amount of deformation subjected to the scent bitmap by the attack; the qualification process is described as follows:

Step 1: For each region calculate actual density of black pixels using:

$$
=\sum_{x=1}^{48} \sum_{y=1}^{48} D s_{i}=D s_{i}+R g n_{x, y}
$$

Where: $D s$ is the density of region $i$, and $R g n$ is the region being processed

Step 2: For each region in qualification stripe calculate standard density threshold using:

$$
\text { stripe region } n_{i}=2 x \sum_{x=1}^{24} \sum_{y=1}^{24} \text { Std }_{i}+\text { Stds } s_{i}+\operatorname{Srgn}_{(x, y)}
$$

Where: Stds is the stripe density of stripe region $i$, and Srgn is the stripe region being processed.

Step 3: Map each density value acquired from the regions to the closest value acquired from the qualification stripes. 
Step 4: Insert the qualified values to the scent values array of the olfactory based Image viewer.

\subsection{Firmware}

The firmware of the olfactory display uses a USB protocol based communication system with 64 byte transfer rate, the main data sent via USB is the actual information about the scent element will be activated and how long it will activate, other supporting data such as fans speed and synchronization data are also sent via USB.

The proposed olfactory display takes the six scent information values and starts activating each scent element accordingly, keeping in mind that maybe more than one scent element might be activated at the same time to mix two or more scent materials, to achieve the intensity, values received at the olfactory display, the value will be decreased by one each iteration while examining the values repeatedly, if the value reaches to zero "no output" then the decrement stops for that scent element deactivating the corresponding scent element, the process is repeated until all the values are exhausted the following steps describe the process:

Step 1: Loop until all values are exhausted

Step 2: For each scent element value

If value $>0$ then

Decrement

Send signal to scent element

End if

Step 3: Wait for a predefined time.

The maximum delay of the highest intensity should not exceed frame duration because the next frame data will be received afterwards.

\section{Experimental Results}

The experimental results were obtained with some parameters to obtain the described results; an MQ-3 sensor is used to detect the output of the olfactory display to measure the speed of the output and the intensity level of the scented material. Using ethanol as a detecting material diluted into water with different concentrations to find the effect of concentration with respect to detection time.

Also, in the experimental results some attacks were applied along with a number of deformations to the scent bitmap to test the resulting values being extracted. In each case the values yielded very good results and where decoded successfully, according to the following test results, a very cohesive correlations between the region data and strip threshold reference data see Figure 9-11.

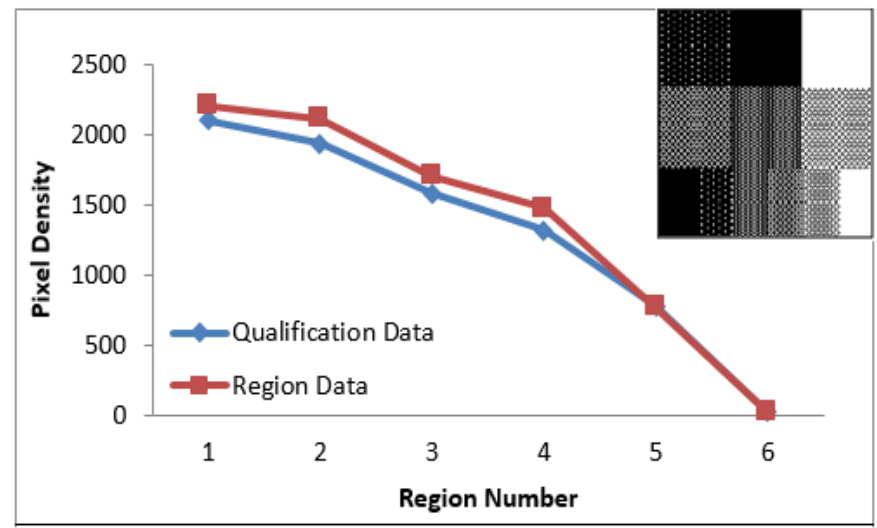


Figure 9. The Extracted Scent Bitmap with no Data Corruption and the Correlation of the Qualification Data with the Region Data Extracted from an Image

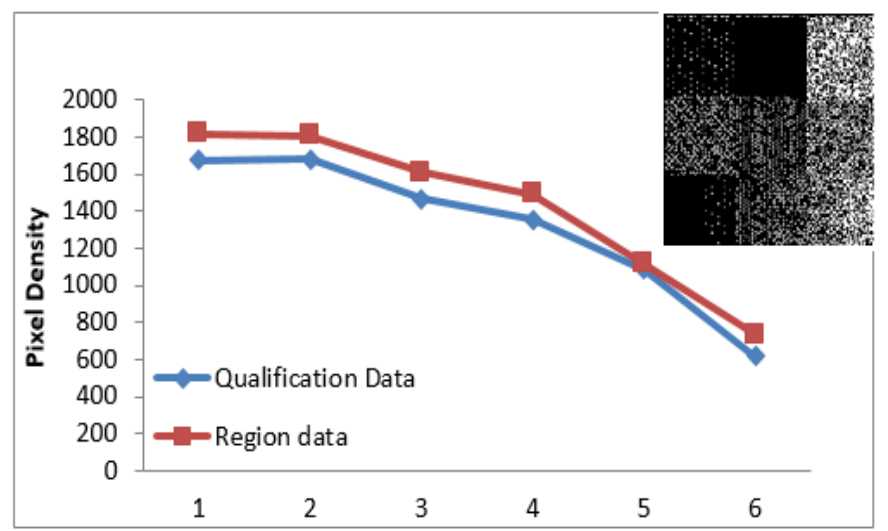

Figure 10. The Extracted Scent Bitmap with Moderate Data Corruption and the Correlation of the Qualification Data with the Region Data Extracted from an Image showing same Correlation with the Original Image

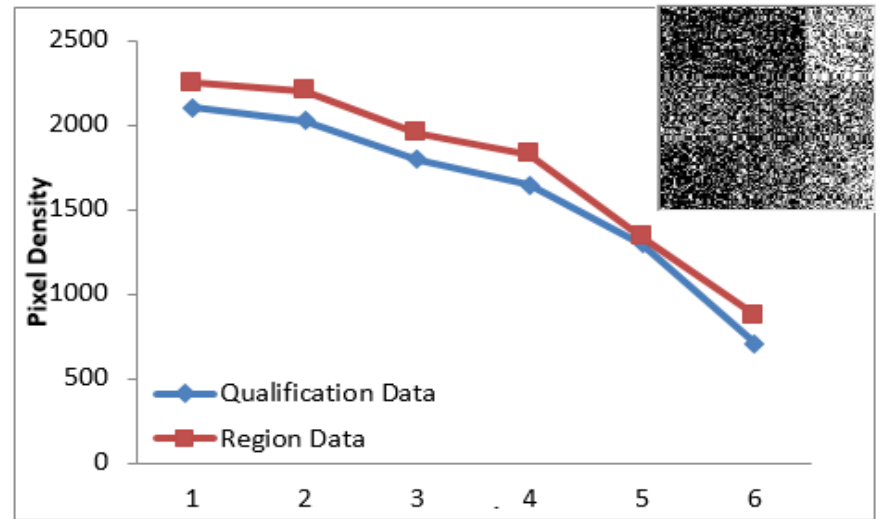

Figure 11. The Extracted Scent Bitmap with Severe Data Corruption and the Correlation of the Qualification Data with the Region Data Extracted from an Image Showing the Same Correlation to the Original Image

The proposed algorithm is applied to four image samples 'test1' through to 'test4', all images are 1920×1080 in resolution and have 24-bits color depth. The scent bitmap image is a binary image of size $144 \times 144$ that contains the scent information is embedded into the image using the algorithm described in the previous section.

In performed experiments, the scaling factors should be between $0.9-1.9$, and the best result is obtained for $\alpha=1.8$, and $\beta=1.5$ to obtain a tradeoff between imperceptibility and robustness. The PSNR lies between $68-70 \mathrm{~dB}$ for the test images, these values indicate that the visual degradations are acceptable and highly imperceptible for HVS. Figure 12 illustrate the plots of PSNR versus image names after embedding process. The performance of the algorithm has been measured in terms of its imperceptibility and robustness against salt and pepper noise, Gaussian noise with mean=0 and variance $=0.0005$, histogram equalization, Gamma Correction 1.5, Intensity Adjustment ([0 0.8], [ [ 1 1]), JPEG compression (with quality factor $=50$ ), and Gaussian low pass filter, see Figure 13, Figure 14, Figure 15 and Figure 16. In Gaussian low pass filter of sizes $9 \times 9,5 \times 5,3 \times 3$, for all images, the $\mathrm{NC}$ is equal to 1 , and with size $2 \times 2$, the $\mathrm{NC}$ is between 0.79-0.84, and finally Figure 17 shows the effect of attacks on PSNR values. 


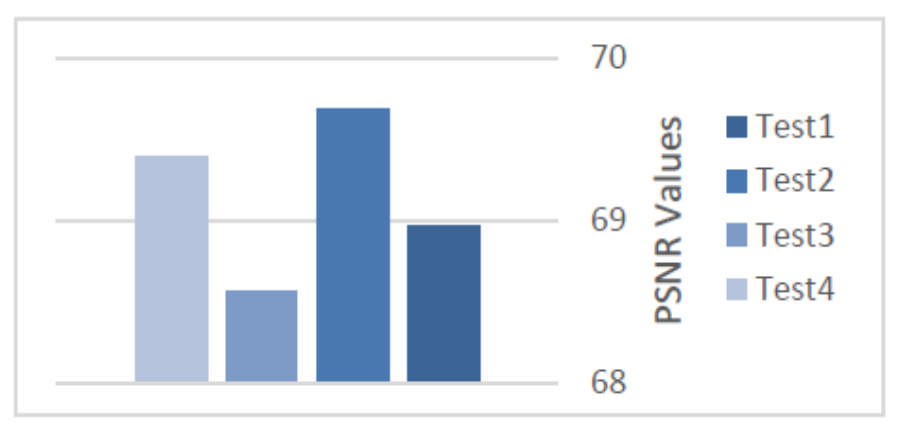

Figure 12. The PSNR Versus Image Names After Scent Bitmap Embedding

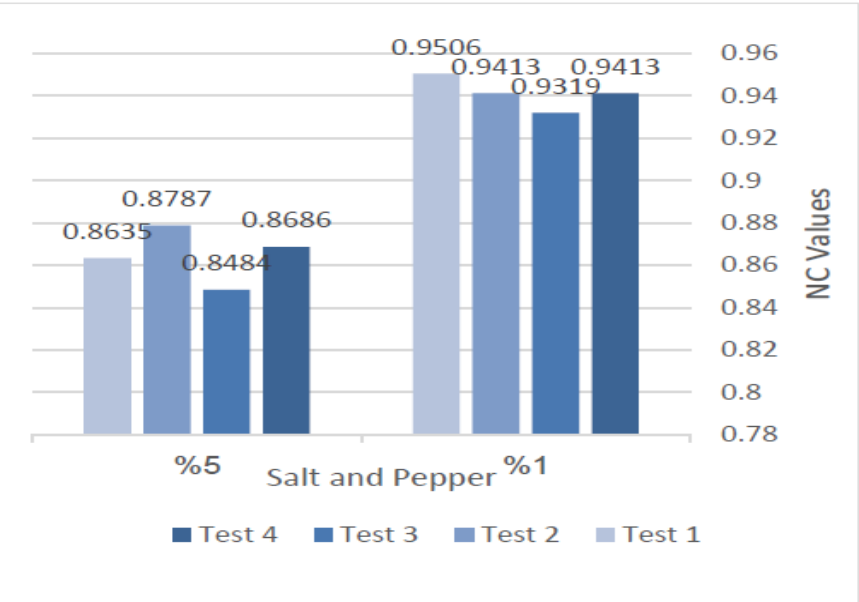

Figure 13. NC Values for Reconstructed Scent bitmap Under Salt and Pepper Noise 1\% and Salt and Pepper Noise 5\%

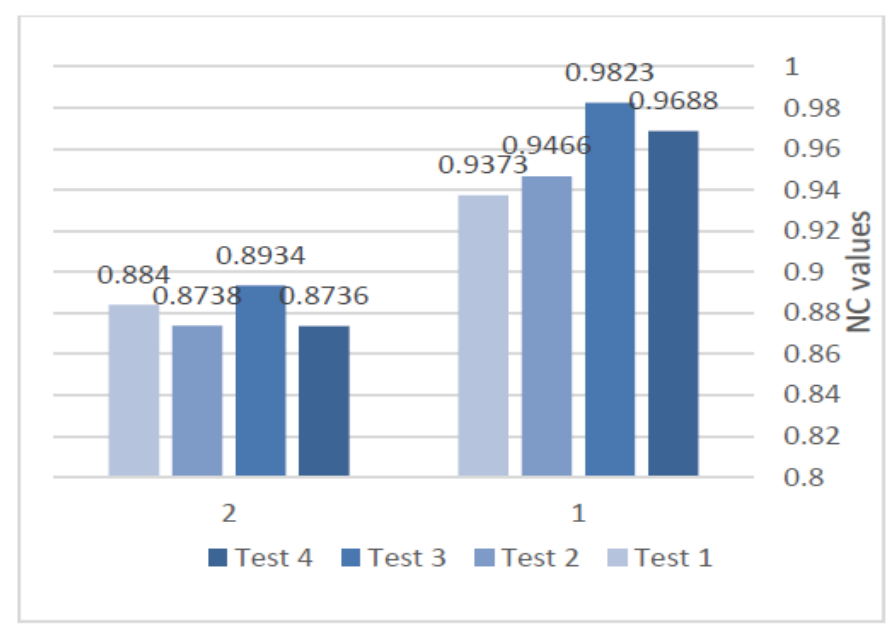

Figure 14. NC Values for Reconstructed Scent Bitmap Under (1) Histogram Equalization and (2) Gaussian Noise 


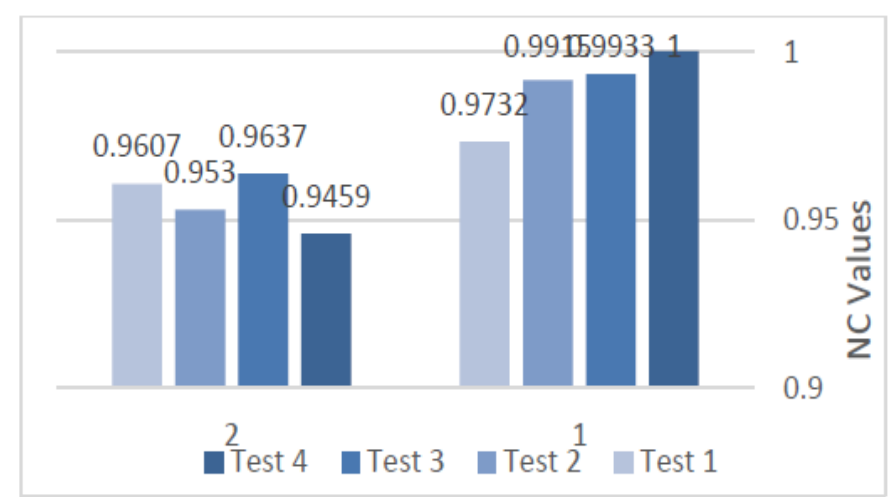

Figure 15. NC Values for Reconstructed Scent Bitmap Under (1) Intensity Adjustment and (2) Gamma Correction

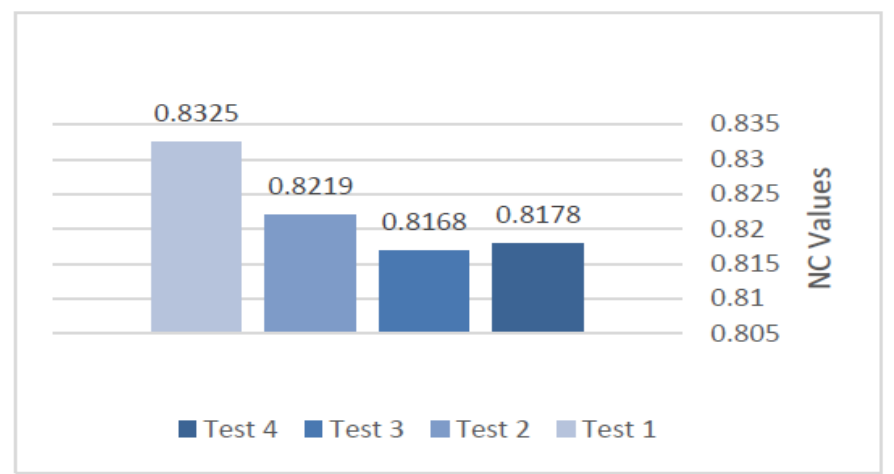

Figure 16. NC Values for Reconstructed Scent Bitmap Under JPEG Compression

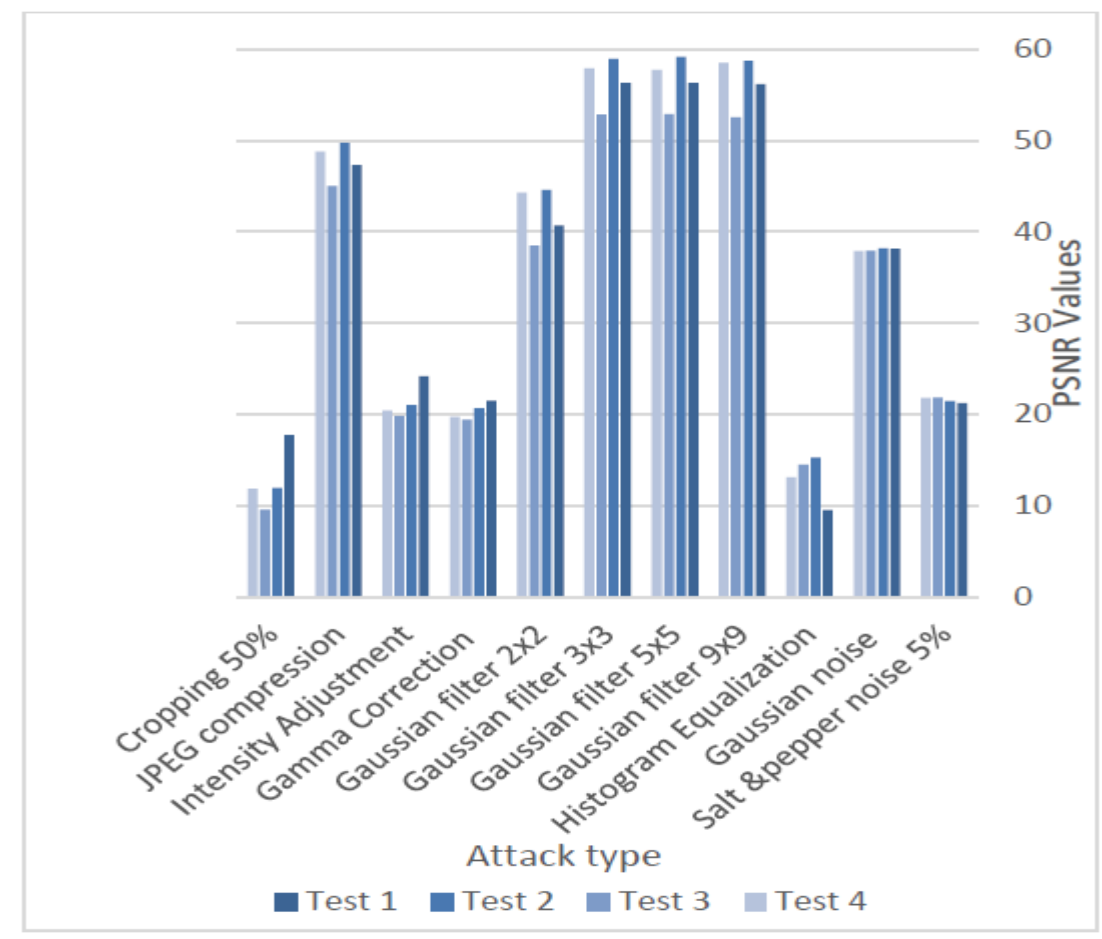

Figure 17. The Effect of Attacks on PSNR Values

\section{Conclusions}


In conclusion, from implementing the proposed olfactory display, it was very clear that using piezoelectric micro-porous film gave very good results in term of response time and intensity control of the amount and state of the ejected scent material, which these three factors are very important in the olfactory displays implementation satisfying our claims in section 2, in addition the low voltage implementation resulted in a device dose not requires an external power supply rather than that, an internal Li-Ion battery was used as a backup for the system which is needed in rare cases of the proposed olfactory display operation.

Also using the scent bitmap in the proposed design provided a very robust implementation to carry out the data embedding and was very immune to sever deformation and data corruption, the qualification strip was in some sense mandatory requirement because a successful qualification threshold depended on such additional information, otherwise the extracted data where quantified successfully but could not be mapped accurately due to the absence of guiding data from the qualification stripe. In addition, the embedding process provided a high capacity, immunity and efficiency in the embedding technique, and was able to hold data much more than needed for the proposed system, indicating that a possible expansion for the technique is feasible to hold more data for even more scent elements to implement comprehensive olfactory displays.

Furthermore, exploiting the blue channel of the image provided an imperceptible data embedding method to avoid affecting the image data since the blue color is hard to perceive its impurities by the HVS.

In the implementation, a large common chamber that all sent elements share is very important for mixing scented material before ejection to be sensed by the human olfactory system as a mixture rather than a number of varying odors through time, finally the system implementation provided a low level of noise as measured in total which less than $55 \mathrm{~dB}$ which hardly noticeable by HAS sitting within 0.5 meter from the olfactory display which is a very important feature in implementing peripheral devices.

Also, according to the obtained results of NC and PSNR values that shows promising results of a fair tradeoff between imperceptibility and robustness proving that the proposed technique describes effective data embedding scheme for merging images with scent information for the proposed olfactory display.

\section{Acknowledgment}

This work was partially supported by the National Natural Science Foundation of China (Grant No. 61173107), the National High Technology Research and Development Program of China (Grant No. 2012AA01A301-01), the Special Project on the Integration of Industry, Education and Research of Guangdong Province, China (Grant No. 2011A091000027) and the Project on the Integration of Industry, Education and Research of Huizhou, Guangdong Province, China (Grant No. 2012C050012012).

\section{References}

[1]. D. W. Kim, H. C. Yeong, K. Nishimoto, Y. Kawakami, S. Kunifuji and H. Ando, "Development of Aroma-Card Based Soundless Olfactory Display", 16th IEEE International Conference on Electronics, Circuits, and Systems, Yasmine Hammamet, Tunesia, (2009) December, pp. 703-706.

[2]. Y. Ariyakul and T. Nakamoto, "Olfactory Display Using a Miniaturized Pump and a SAW Atomizer for Presenting Lowvolatile Scents”, IEEE Virtual Reality Conference, Singapore, (2011) March, pp. 193194.

[3]. T. Yamanaka, R. Matsumoto, and T. Nakamoto, "Study of odor blender using solenoid valves controlled by delta-sigma modulation method for odor recorder", Sensors and Actuators B, Chemical, vol. 87, (2002) August, pp. 457-463.

[4]. T. Nakamoto and K. Yoshikawa, "Movie with scents generated by olfactory display using solenoid valves", IEICE Trans. Fundamentals, vol. E89-A, no. 11, (2006), pp. 3327-3332. 
[5]. T. Nakamoto, N. Nimsuk, B. Wyszynski, H. Takushima, M. Kinoshita and N. Cho, "Reproduction of scent and video at remote site using odor sensing system and olfactory display together with camera, IEEE SENSORS, lecce, Italy, (2008) October, pp. 799-802.

[6]. J. Waleed, H. D. Jun, S. Hameed, H. Hatem and R. Majeed, "Integral Algorithm to Embed Imperceptible Watermark into Anaglyph 3D Video", International Journal of Advancements in Computing Technology (IJACT), vol. 5, no. 13, (2013), pp.163-173.

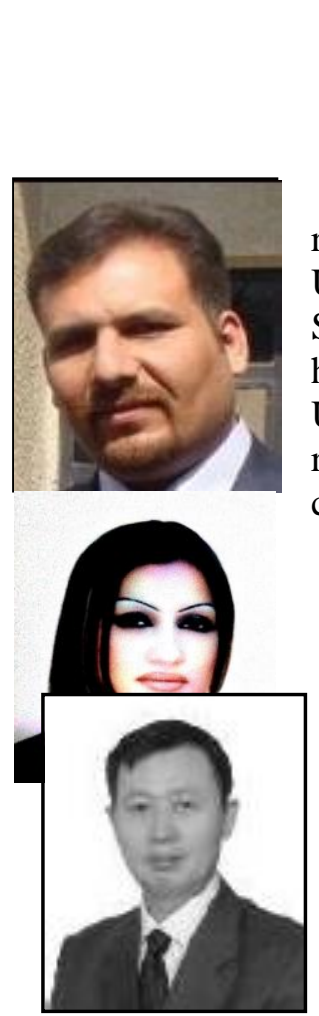

Saad Hameed was born in Baghdad - Iraq in 7 June 1979, he received his B.Sc. Degree in computer science at AL-Mansour University College 2001, and Masters Degree in computer Sciences, Iraqi committee for computer and informatics in 2004, he continued working in academic teaching in AL-Mansour University College for 11 years, through that time all of his research was self-funded and concentrated in automation and control, he has been promoted from assistant Lecturer to Lecturer in 2011, he is now a Ph.D. degree student at Hunan University in P.R. China.

Zhiyong Li received the MSc degree in System Engineering from National University of Defense Technology, Changsha, China, in 1996 and PhD degree in Control Theory and Control Engineering from Hunan University, Changsha, China, in 2004. Since 2004, he joined the College of Information Science and Engineering of Hunan University. Now, he is a Full Professor with Hunan University, member of China Computer Federation $(\mathrm{CCF})$. His research interests include visual object tracking, embedded computing system, dynamic multi-objective optimization evolutionary algorithm and tasks scheduling optimization in cloud computing. He has published more than 50 papers in international and domestic journals and conferences.

Prof. Li obtained several awards from academic organizations and conferences, such as the Champion of the Future Challenge: Intelligent Vehicles and Beyond, FC'09, which was hosted by the National Natural Science Fund Committee of China in 2009.

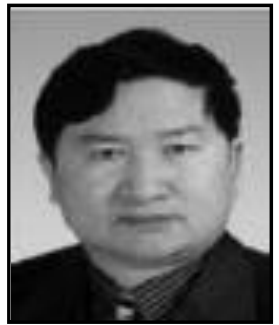

Renfa Li was born in Hunan - China in 1957, he works as Professor and $\mathrm{PhD}$ supervisor of Hunan University, Senior member of China Computer Federation (CCF), his main research interests include embedded system, artificial intelligence and optimization theory etc. 


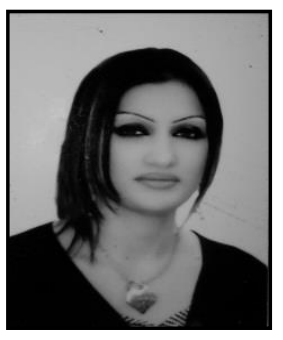

Jumana Waleed is a Ph.D. student in the School of information science and Engineering at Central South University, Changsha, China. Her research activity focuses on image processing, and information security working on digital watermarking. She received the B.S. degree in computers sciences from the Al-Yarmouk University College, Iraq, in 2004, and the M.S. degree in Computer Science/Data Security from the University of Technology, Baghdad, Iraq, in 2009. 
International Journal of Control and Automation Vol. 8, No. 2 (2015) 